UJMR, Volume 6 Number 2, December, 2021, pp 130 - 134

ISSN: $2616-0668$

https://doi.org/10.47430/ujmr.2162.018

Received: $15^{\text {th }}$ November, 2021 Accepted: $31^{\text {st }}$ December, 2021

\title{
Menace of Ebola Virus Disease: A Review
}

\author{
${ }^{* 1}$ Galadima, M.A., ${ }^{2} \mathrm{Ali}, \mathrm{J}$. and ${ }^{2} \mathrm{Garba}, \mathrm{K}$. \\ ${ }^{1}$ Community Health Department ${ }^{2}$ Medical Laboratory Science Department \\ College of Health Sciences and Technology P.M.B 1028, Nguru Yobe State Nigeria \\ "Corresponding Author: abufusam83@gmail.com; Tel: +234-7034977924
}

\begin{abstract}
Ebola Virus Disease (EVD) a deadly disease that affect human and apes like gorilla, chimpanzee antelopes and so on. The causative virus is found mostly in Africa. It had been uncovered first in mid 1970s on the brink of the River of Ebola village. Since from the primary inception of the strange disease in Democratic Republic of Congo, there has been a periodic outbreak of the disease in some African countries, affecting many people within the region. A systematic review was conducted using Google search Engine, Cochrane database systemic review (CDSR), Centre for Disease Control and prevention and World Health Organization EVD report Databases. The 2014-2016 Ebola outbreaks in West Africa were the most important and most complex, which began in Guinea and spread to Liberia, Nigeria and Sierra Leone. Before the disease is contained, it kills about 11,000 people and quite 28,000 people being affected from it first mid 1970s detection to late 2016. The virus is transmitted from fruit bat in touch of with other animals and passes on the infection to humans through handling infected, dead or sick animals found within the forest or through direct contact with bats. Human to human transmission occurs through direct contact with the blood, secretions and other body fluids. Symptoms of the virus are: sudden onset of fever, fatigue, muscle pain, headache, sore throat, vomiting, diarrhea, rash, impaired kidney and liver function, and bleeding from body openings. Recent advancements have been carried out in the form of effective Ebola Virus Vaccine Inmazeb and Ebanga for Zaire Ebola Virus and anti-Ebola virus drug rVSV-ZEBOV (Ervebo). However, the rapid geographic propagation, non-specific clinical presentation, lack of adequate vaccine and specific diagnostic assay are the possible challenges to combat the dreaded public health menace of Ebola Virus Disease.

Key Words: Ebola Virus, Disease, Transmission, Treatment, Vaccine
\end{abstract}

INTRODUCTION

Ebola virus diseases (EVD), formerly referred as Ebola Hemorrhagic fever (EHF), may be a rare but severe, often mortal illness in humans (WHO, 2021a). Ebola may be a deadly disease caused by an epidemic, which is transmitted from animals to humans, then from humans to other humans (Nabil, 2014) The average Ebola Disease case lethal rate is estimated to 50 per cent, with rates differed from 25 per cent to 90 per cent in past epidemics (WHO, 2021b). The virus causes a quick, serious illness which is usually mortal if untreated. Ebola virus disease (EVD) primarily emerged in 1976 in two (2) simultaneous epidemics, one in what is now Nzara, South Sudan, and the other in Yanbuku, Democratic Republic of Congo (DRC) in a brink of Ebola River, from which the disease takes its name ("Sino", 2021; WHO, 2021a). The disease is characterized by wide spread petechial hemorrhages, focal necrosis of the kidney, spleen and liver, shock and ultimately death ("Sino", 2021). The Ebola virus spreads through contact with the body fluids like vomits, faeces or blood of an infected person, or through surfaces and materials like bedding, clothing contaminated with these fluids (WHO, 2021b). The incubation time, that is the time intervals from infection with the virus to onset of symptoms, is from 1-3 weeks. The symptoms of EVD are often sudden and include fever, fatigue, muscle pain, headache and sore throat. This is often followed by vomiting, diarrhea, rash, symptoms of impaired kidney and liver function and in some cases internal and external bleeding (WHO, 2021b). The 20142016 epidemics in West Africa were the most important Ebola epidemic since the virus was primarily detected in 1976. The epidemic started in Guinea and spread across land borders to Sierra Leone and Liberia (WHO, 2021a).

\section{MATERIALS AND METHODS}

The study review results of publications on all Ebola Virus outbreaks. Information was obtained from websites of World Health Organization, Centre for Disease Control and prevention, Food and Drug Administration and Cochrane Library, and some articles were also taken from Google scholar. Abstracts and full texts were screened by the reviewers in 
UJMR, Volume 6 Number 2, December, 2021, pp 130 - $134 \quad$ ISSN: 2616 - 0668

selecting relevant articles based on the inclusion criteria. The review focus on the largest studies and the most recently published study results at the time of this review (AugustNovember, 2021). Studies were selected to cover major Ebola endemic affected countries.

\section{Brief History of Ebola Virus Disease}

The Ebola Virus Disease was discovered first in 1976, during an outbreak of Ebola hemorrhagic fever that occurred in Zaire and later in Sudan in that time. There were about 300 victims in each of the outbreak. The first person affected with Zaire Ebola Virus Disease was a Village head Teacher Mabalo Lokela by name, who stated displaying sign and symptoms of the strange disease on August, 261976 (Makund et al., 2015). Tai Forest disease formally referred to as Ivory Cost Ebola virus Disease was discovered in 1994 by a Scientist conducting autopsies on Monkey and contracted the virus. Zaire Ebola virus Disease second outbreak occurred in 1995 in which 315 were affected and 254 people were death (Makund et al., 2015).

Makund et al. (2015) also reported that in the year 2000, Uganda had an outbreak of Ebola Virus which affected and killed 425 and 224 respectively and Sudan Ebola virus was responsible. There was another outbreak in Democratic Republic of Congo in 2003 that affected 143 and killing 128 people. In 2007, new species of the virus surface in Uganda named Bundibugyo affecting 149 and killing 37 people (Makund et al., 2015). In March, 2014 World Health Organization (WHO) reported an outbreak of Ebola virus Disease in Guinea, which the researchers traced to a 24 months old child that died in the year 2013. It was rapidly spread to other countries like Liberia, Nigeria and Sierra Leone. This outbreak was the largest and most fatal outbreak ever recorded in the region affecting about 28,000 people and killing about 11,000 people (Makund et al., 2015; WHO, 2014).

\section{Ebola Virus Disease in Nigeria}

During the West African Ebola virus epidemic in July 2014, an infected person LiberianAmerican man, Patrick Sawyer Flew from Monrovia, Liberia, to Lagos, Nigeria, brought EVD into Nigeria. Exposed responders were afterwards infected. To ward off a quick spread within Africa's most populous city, prompt reaction efforts concentrated on quickly establishing Ebola treatment units, training caregivers, and identifying all contacts for each case of Ebola. As a result of this rapid response, the outbreak was confined to two cities, Lagos and Port Harcourt, and additional spread of the Ebola virus through Nigeria and into other areas in Africa was prevented. WHO declared Nigeria Ebola free on October 20, 2014 (WHO, 2014).

Mode of Transmission of Ebola Virus Disease The Ebola Virus Disease affects people living near tropical rain forest. The affected people are mostly isolated and the people they have been in contact with should be tracked and monitored for three weeks for clinical symptoms of the disease. Scientist think people are initially infected with Ebola virus through contact with infected animals, such as a fruit bat or non-human primates this is called spillover event. Subsequently, the disease spread from human to human, potentially infecting a versed number of human being (Beth et al., 2016; CDC, 2021a). This virus spreads through direct contact (like through broken skin or mucous membranes in the eye, mouth or nose) with: Blood or other body fluids (Urine, Sputum, Stool, Sperm, Breast Milk, Sweat, Amniotic fluid and Vomit) of a person who is ill with or has died from Ebola virus disease (EVD). Things (such as Cloths, Needles Bedding and Medical care Equipments) contaminated with body fluids from a person who is ill with or has died from (EVD) Infected fruit bats or non-primates (like Apes, Antelopes and Monkeys) Semen from a man who recovered from EVD through oral, vaginal or anal sex. The disease may be left in some certain body fluids like sperm of a patient who has regained from Ebola Disease, albeit they are doing not to have signs of severe illness. There is no proof that Ebola disease can be spread during sex or getting in touch with vulva fluids from a woman that has had Ebola disease (CDC, 2021a). By the time human being becomes infected with Ebola disease, they do not start showing clinical symptoms instantly. The interval between exposure to pathogen and developing symptoms is termed the incubation period. Human being can only spread Ebola disease to others after developing clinical and symptoms of Ebola disease (Beth et al., 2016; CDC, 2021a).

\section{Symptoms of Ebola Virus Disease}

Symptoms may appear anywhere from 2-21 days after contact with the virus, with an average of 7-10 days. The course of the illness typically progresses from "dry" symptoms initially (like fever, aches and pains, and fatigue), and then progresses to moist signs of the disease which often includes: Fever, Aches and pains, like intense muscle and joint pain, headache and abdominal (stomach) pain, Weakness and fatigue, Gastrointestinal symptoms including diarrhea and vomiting, Unexplained hemorrhaging bleeding or bruising (Beth et al., 2016; CDC, 2021a; Nabil, 2014). 
UJMR, Volume 6 Number 2, December, 2021, pp 130 - $134 \quad$ ISSN: 2616 - 0668

Other symptoms may include red eyes, skin rash, and hiccups (late stage) (CDC, 2021b). WHO (2021a) reported that symptoms of Ebola virus in some cases, there is both the internal and external bleeding (for example oozing from the gums, or in the stool). So many mutual illnesses may have exerted clinical symptoms as Ebola Disease, including typhoid fever, malaria, tuberculosis or influenza (flu).

\section{Diagnosis of Ebola Virus Disease}

It is often difficult to clinically distinguish Ebola virus Disease from other communicable disease like Malaria, Typhoid fever and Meningitis. Many symptoms of pregnancy and Ebola disease are also quite similar because of risk to the pregnancy; pregnant women should ideally be tested rapidly if Ebola is suspected (WHO, 2021a) Confirmation that symptoms are caused by Ebola virus infection are made using the subsequent diagnostic methods: Antibodycapture Enzyme- Link Immunosorbent Assay (ELISA), Antigen-capture Detection test- Serum neutralization test, Reversed Transcriptase Polymerase Chain Reaction (RT-PCR) Assay, Virus Isolation by cell Capture and Electron Microscopy (WHO, 2021a).

\section{Who are at risks of contacting Ebola Virus Disease?}

Health care workers who do not use proper infection control while caring for Ebola patients and family and relatives in touch with infected patients, are at the very best danger of getting ill. Ebola disease may be transmitted when people come in touch with infected blood or other related fluids. The Ebola disease only poses a little danger to travelers or the public who did not cared for or been in touch (within 3 feet or 1 meter) with someone infected with the disease (CDC, 2021b).

\section{Persistence of Ebola Virus Disease}

The disease can stay in parts of the body that are immune opportune sites after a short infection. These are sites where viruses and pathogens, like the Ebola disease are covered from the survivor's system, even after being cleared elsewhere in the body. These areas include the testis, aqueous humor of the eyes, breast, placenta, and central nervous system, particularly the cerebrospinal fluid (CDC, 2021b). Whether the virus is present in these body areas and how long varies by survivors, researchers are now probing how long the virus remains in these body fluids within Ebola disease survivors (CDC, 2021b).

\section{Mode of Prevention of Ebola Virus Disease} When living in or travelling to a neighborhood where Ebola virus is potentially present, there are a variety of ways an individual can use to guard himself and stop the spread of Ebola virus disease. Avoid contact with blood and body fluids (like Urine, Feces, Saliva, Sweat, Vomit, Breast Milk, Amniotic Fluids, Semen, and Vaginal Fluids) of people who are sick. Avoid contact with semen from a man who has recovered from EVD until laboratory testing shows that the virus is gone from his semen Avoid contact with items which will have come in touch with an infected person's blood or body fluids (like Cloths, Bedding, Needles and Medical Equipments). Avoid funeral or burial practices that involve touching the body of someone who died from EVD or suspected EVD. Avoid getting in touch with Bats, apes, Forest Antelopes, and non-human Primates (e.g. Monkeys, and Chimpanzees) blood, other bodily fluids or uncooked meat prepared from these or unfamiliar animals (bush meat) (CDC, 2021b).

\section{Ebola Virus Vaccine}

CDC (2021a) reported that the United State Food and Drug Administration (FDA) approved the Ebola Vaccine rVSV-ZEBOV (called Ervevo ${ }^{T M}$ ) on December 19, 2020 This is the first FDAapproved vaccine for Ebola virus disease. This vaccine is given as a one dose vaccine and has been found to be safe and protective against Zaire Ebola virus, which has caused the most important and most deadly Ebola outbreak to date. On February 26, 2020, the Advisory Committee on Immunization Practices (ACIP) suggested pre-exposure preventive vaccination with rVSV-ZEBOV for adult's $\geq 18$ years age within the U.S population who are at potential occupational risk of exposure to Zaire Ebola disease. This recommendation includes adults who are: Responding or getting to answer an epidemic of Ebola virus Disease, Laboratories or other staff working at biosafety facilities that work with live Ebola virus, Healthcare personnel working at federally designated Ebola treatment centers in countries. A two-dose vaccine regimen of a different vaccine that was also designed to protect against the Zaire Ebola virus species Ebola- was used under a research protocol in 2019 during an Ebola outbreak within the Democratic Republic of Congo. The two doses of this vaccine used two distinct vaccine components (Ad 26. ZEBOV and MVABN-Filo) and the regimen requires an initial dose and a 'booster' dose 56 days after. This vaccine is not yet endorsed by the Food and Drug Administration for ordinary use (CDC, 2021b).

WHO (2021b) recounted that, the Ervebo vaccine had been shown to be efficient in protecting human being from the kind of Zaire Ebola disease, and is recommended by the strategic advisory group of experts on 
UJMR, Volume 6 Number 2, December, 2021, pp 130 - $134 \quad$ ISSN: 2616 - 0668

immunization as part of broader set of Ebola outbreak response tools. In December, 2020, the vaccine was endorsed by the U.S Food and Drug Administration and prequalified by World Health Organization for use in individuals 18 years of age and older (excepts for pregnant and breast feeding women) for protection against Ebola virus Disease caused by Zaire Ebola virus. WHO (2021b) further reported that the vaccine had been administered to more than 350,000 people in Guinea and in the 20182020 Ebola virus disease outbreaks in the Democratic Republic of Congo under "Compassionate use" principle. The vaccine has shown to be safe and effective against the species of Zaire Ebola Virus. A global stock pile of the Ervebo vaccine has been available starting January, 2021 (WHO, 2021a). In May 2020, the European Medicines Agency recommended granting marketing authorization for a 2-component vaccine called Zebdeno and Mvabea for individuals 56 weeks upward. The vaccine is taken in 2 doses: Zebdeno is taking first followed by Mvabea which is given in 8 weeks later as a second dose. This prophylactic 2-dose regimen is therefore not suitable for an outbreak response where immediate protection is necessary (WHO, 2021a).

\section{Treatment of Ebola Virus Disease}

CDC (2021b) reported that, there are currently two treatments approved by the U.S. Food and Drug Administration (FDA) to manage EVD posed by the Ebola disease, species Zaire Ebola virus, within adults and children. The primary drug approved in October, 2020. Inmazeb may be a combination of three monoclonal antibodies (Atoltivimab, Maftivimab, and Odesimabebgn). The other drug, Ebanga, may be a single monoclonal antibody that was endorsed in December, 2020. Monoclonal antibodies (often abbreviated as mAbs) are proteins produced in a laboratory or other manufacturing facilities that act like natural antibodies to prevent a germ like an epidemic from replicating after its infected an individual. These particular mAbs bind to a part of the Ebola disease's surface known as glycoprotein, which stop the virus from entering a person's cells (CDC, 2021b). In a nut shell Inmazeb targets the glycoprotein that is on the surface of Ebola disease. Glycoprotein binds to the cell receptor and fuses the viral and host cell membranes allowing the virus into the cell. The three antibodies that structure Inmazeb can bind to the present glycoprotein simultaneously and block attachment and entry of the virus into the cell. Ebanga blocks binding of the virus to cell receptor, preventing its entry into the cell. Both of these managements, alongside two others, were evaluated in a randomized controlled trial during the 2018-2020 Ebola epidemics within the Democratic Republic of Congo. The overall survival was much higher for patients receiving either of the two drugs that were now endorsed by the FDA. Neither Inmazeb ${ }^{\mathrm{TM}}$ nor Ebanga $^{\mathrm{TM}}$ are evaluated for efficacy against species apart from Zaire Ebola disease (CDC, 2021b).

\section{Supportive care to Ebola Virus Patients}

CDC (2021b) Reported that whether or not other treatments are available, basic interventions are often significantly improve chances of survival when provided early. These are mentioned as supportive care, and include: Providing fluids and electrolytes (body salt) orally or through infusion into the vein (intravenously). Using medication to support vital sign, reduce vomiting and diarrhea, and to manage fever and pain, testing other infections, if they occur. Furthermore WHO (2021a) reported that rehydration with oral or intravenous fluids and treatment of specific symptoms improved survival. A range of potential treatments includes blood products, drug therapies and immune therapies are currently being evaluated.

\section{Controlling Infection in Healthcare Settings} Medical workers must take necessary standard preventive measures when caring for patients, no matter their presumed assayed result. The measures may include basic proper hand hygiene, respiratory hygiene, use of Personal Preventive Equipment (to block spills or other in touch with infectious materials), safer injection and burial skills. Healthcare workers caring for patients with suspected or confirmed Ebola virus should apply extra infection control measures to stop contact with the patient's blood and body fluids and contaminated surface or materials like clothing and bedding. When in touch (within a meter) of patients with the disease, medical personnel most use a face mask (a face protector or an eye goggles), a clean, laboratory coat, and disposable hand gloves (sterile gloves for some procedures) (WHO, 2021b).

\section{Care for People who recovered from Ebola Virus Disease}

A number of medical complications have been reported in people who recovered from Ebola, including psychological state issues. Ebola virus may persist in some body fluids, including semen, pregnancy related fluids and breast milk (WHO, 2021b). All Ebola virus survivors and their sexual partners should receive counseling to ensure safer sexual practices until their semen has twice tested negative. Survivors should be giving condoms as a contraceptive or 
UJMR, Volume 6 Number 2, December, 2021, pp 130 - $134 \quad$ ISSN: 2616 - 0668

as a way to prevent the spread of the Ebola disease through sex. Male Ebola survivors should be offered semen testing at 3 months after onset of disease, then for those that test positive, monthly thereafter until their semen tests negative for virus twice by RT-PCR, with an interval of 1 week between tests. Ebola survivors and their sexual partners should either abstained from all types of sex or observed safer sex through correct and consistent condom use until their semen has twice analyzed negative. The survivors may continue normal intimacy practice when their sperm assay shows negative.

WHO suggested that male who recovered from Ebola disease may practice safe sex and hygiene for 12 months from onset of symptoms

\section{REFERENCES}

Beth, P.B., Inger, K.D., Daniel, B.J., Thomas, A.K., Sturt, T.N., John, P.O.C and Jordan, W.T

.(2016). Over view, Control Strategies \& lesson learned in the CDC Response to the 2014-2016 Ebola epidemics. MMWR/July 8, 2016/ Vol. 65/No.3 US Department of Health and Human Service/ Center for Disease Control and Prevention

Center for Disease Control \& Prevention (2021a) Morbidity \& Mortality Weekly Report (MMWR) over view, Control Strategies \& lesson learned in the $C D C$ response to the 2014-2016 Ebola epidemic. Supplements/July 8, 2016165 (3); 4-11. Accessed 2/5/.2021

Center for Disease Control \& Prevention (2021b) Ebola outbreak in West Africa/History/Ebola (EVD). www.cdc.gov/vhf/ebola/history/20142016-outbreak/index.html Accessed 2/5/.2021

Makund, J., Rajesh, P., Kuldip, S. and Jasbir, S. (2015). The Ebola Menace: Epidemic and

Expectations International Journal of current Microbiology and Applied Sciences Volume 4 Number 1 (2015) Pp $731-747$

Nabil Ebrahiem (2014). YouTube Educational animated video with English subtitles.describes the Ebola Virus Disease and how it is spread. Retrieved on $2 / 5 / .2021$ from or until their semen tests negative twice for Ebola virus (WHO, 2021b)

\section{CONCLUSION}

Ebola Virus Disease has emerged as a big global public health menace due to multiple disease outbreaks in the last 3-4 decades. Recent advancements have been carried out in the form of effective Ebola Virus Vaccine Inmazeb \& Ebanga for Zaire Ebola Virus \& anti-Ebola virus drug rVSV-ZEBOV (Ervebo). However, the rapid geographic propagation, non-specific clinical presentation, lack of adequate vaccine \& specific diagnostic test are the possible challenges to combat the dreaded public health menace of Ebola virus Disease.

http://www.youtube.com/drebraheim

Sino (2021): What is Ebola Virus Disease? Sino online. Retrieved at https: / /www.sinobiological.com/resear ch/virus/what-is-Ebola-virus-vaccine U.S.A on 29/04/2021

World Health Organization (2014) WHO declares end of Ebola outbreak in Nigeria. Media center statement, 20 October, 2014. www.who.int/mediacenter/news/state ments/2014/nigeria-endebola/en/Accessed 2/5/.2021

World Health Organization (2015) Health Workers Ebola Infections in Guinea, Liberia

Sierra Leone: A Preliminary Report 21 May 2015 Accessed 29/04/2021 http://www.who.int/hil/documents/29 105/2015 web final

World Health Organization (2021a). WHO Ebola Virus Disease. Fact sheet. www.who.int/newsroom/factsheets/details/ebola-virusdisease Accessed 29/04/2021

World Health Organization (2021b). News .un .org/en/story/2021/02/1084692 UN News two dies as new Ebola outbreak declared in Southern Guinea Accessed 29/04/2021

World Health Organization (2021c). WHO Africa West African Countries ramp up Ebola preparedness.www.afro.who.int/news/ west-african-countries-ramp-ebolapreparedness cited $2 / 5 / 21$. 